\title{
Laboratory observation and modeling of extreme ultraviolet spectra of highly ionized calcium
}

\author{
V.A. Soukhanovskii ${ }^{1}$, S. Lippmann ${ }^{2}$, M.J. May ${ }^{1}$, M. Finkenthal ${ }^{1,5}$, H.W. Moos ${ }^{1}$, K.B. Fournier ${ }^{3}$, W. Goldstein ${ }^{3}$, \\ D. Pacella ${ }^{4}$, and G. Mazzitelli ${ }^{4}$ \\ 1 Department of Physics and Astronomy, The Johns Hopkins University, Baltimore, Maryland 21218, U.S.A. \\ 22714 Fairview Ave. E. \# 101, Seattle, Washington 98102, U.S.A. \\ ${ }^{3}$ Lawrence Livermore National Laboratories, P.O. Box 808, Livermore, California 94550, U.S.A. \\ 4 Associazione EURATOM-ENEA sulla Fusione, C.R. Frascati, CP 65-00044 Frascati Roma, Italy \\ 5 Permanent address: Racah Institute of Physics, The Hebrew University, Jerusalem, Israel
}

Received October 8; accepted November 23, 1999

\begin{abstract}
Benchmarking and validation of atomic calculations are crucial for understanding the properties of astrophysical and fusion plasmas. An extended re-evaluation of a previous experimental study of the CaXVIII - CaXII extreme ultraviolet (XUV) spectra is presented. $\mathrm{CaF}_{2}$ was introduced into tokamak plasmas and the spectra of the calcium ions were recorded by a photometrically calibrated grazing incidence time-resolved spectrometer. The local plasma electron temperature and density were measured independently. Nearly all features of the line-of-sight integrated spectra were identified. Atomic data for this work were generated ab initio with the HULLAC suite of codes. The results of collisional-radiative (CR) modeling for individual charge states agree with the measured spectral line intensities within the experimental accuracy for most lines, thus validating the electron temperature and density diagnostic potential of the L-shell lines. In addition, we compare experimentally measured and calculated line intensities with those calculated using the CHIANTI database.
\end{abstract}

Key words: atomic processes, Sun: UV radiation, Sun: flares

\section{Introduction}

Extreme ultraviolet (XUV) solar emission lines corresponding to $n=2, \Delta n=0$ transitions of highly ionized calcium, have been recorded in the past by spaceborn spectrometers (OSO-5, OSO-6, Skylab NRL/ATMS082A) and sounding rocket-born instruments (Aerobee

Send offprint requests to: V.A. Soukhanovskii, e-mail: vlad@jhu.edu
150, 200 rockets), and have been used for wavelength identifications, temperature and density estimates in solar flares and for development of solar atmosphere models (Lawson \& Peacock 1984; Feldman et al. 1988; Mason \& Monsignori Fossi 1994 and references therein). The XUV lines emitted by L-shell calcium ions constitute a unique diagnostic tool for studying plasma conditions in the solar flares. This is because many of the spectral line ratios due to $2 \mathrm{~s}^{2} 2 \mathrm{p}^{k}-2 \mathrm{~s} 2 \mathrm{p}^{k+1}$ transitions are density and temperature sensitive, and because of the high formation temperature and density limits of these ions (logarithmic temperatures between 6.25 and 6.75 , electron density range $10^{9} \div 10^{13} \mathrm{~cm}^{-3}$ ). There are few solar abundant elements which provide useful flare diagnostics based on the XUV line ratios in this range of densities and temperatures. Most observed Fe IX - Fe XV XUV line intensity ratios are sensitive to electron density $\leq 10^{11} \mathrm{~cm}^{-3}$ (Dere et al. 1979; Brickhouse et al. 1995), most of the FeXVI - Fe XXIV XUV line ratios are density-sensitive at $n_{\mathrm{e}} \geq 10^{13} \mathrm{~cm}^{-3}$ (Feldman et al. 1992), and their formation temperatures are high. The ions of the third period elements ( $\mathrm{Si}, \mathrm{Mg}, \mathrm{S}$ ) appear to be less abundant in the flares, and their observed lines, useful for $T_{\mathrm{e}}$ and $n_{\mathrm{e}}$ diagnostics, are in the longer wavelength region (above 300 $\AA$ ), inaccessible to a typical grazing incidence XUV spectrometer. Previously, the Skylab NRL/ATM-S082A solar flare observations have been the main source of measured calcium line intensities. Recently flown solar missions (e.g. SERTS, SOHO) have produced a wealth of new spectroscopic data. Its interpretation relies on the accuracy of the available atomic data. High quality spectra of the solar abundant elements, obtained from a laboratory plasma can provide a test for the validity of atomic data and collisional-radiative (CR) models. This is possible because the spectra are less contaminated by emission lines from 


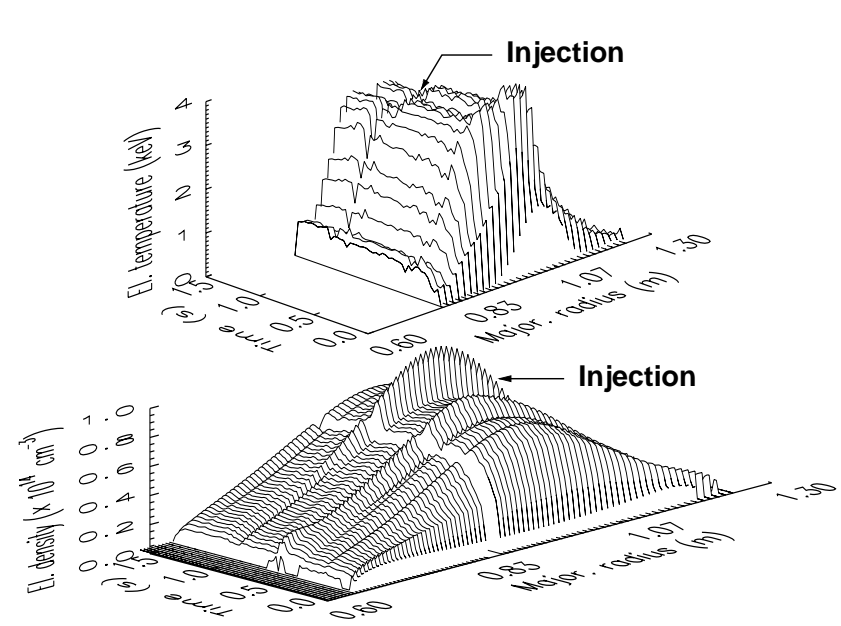

Fig. 1. Time histories of electron temperature (top) and density (bottom) spatial profiles of the FTU tokamak plasma

other elements and local plasma parameters (such as electron density and electron temperature) are independently measured (Finkenthal et al. 1987).

This work presents a re-evaluation of the laboratory study of beryllium- through oxygen-like calcium spectra performed by our group several years ago (Lippmann et al. 1987). The spectra, recorded at the TEXT tokamak (University of Texas, Austin), have been re-analyzed, previously unpublished lines have been added and the analysis was extended to lithium- and fluorine-like charge states. We also analyze new calcium spectra, recorded at the FTU tokamak (Frascati, Italy). In the previous work (Lippmann et al. 1987; Huang et al. 1987), the difficulty in interpreting the experimental results was mainly due to the accuracy of the available atomic data. Atomic transition rates were taken from the literature, or extrapolated from other ions of the same isoelectronic sequence. The complexity of the model, i.e. the types of the processes considered and the number of levels included, was limited. In several cases these factors precluded full analysis of the experimental data. In this work, a CR model, based on ab initio calculated transition rates, is used. For Be I-, B I- and $\mathrm{C}$ I-like calcium ions, a detailed model is constructed in order to study the role of collisional and radiative processes from excited states in application to electron temperature and density diagnostic potential of these ions. We also compare our calculations and measurements with the predictions based on the atomic data from the astrophysical database CHIANTI (v. 2.0, Dere et al. 1997; Landi et al. 1999). CHIANTI contains the best available atomic data for the ions of astrophysical interest and has been used in SERTS and SOHO data analysis (e.g. Young et al. 1978; Mason et al. 1997; Landi \& Landini 1997). Throughout the paper, the units of $\mathrm{eV}$ are used for the temperature $k T_{\mathrm{e}}$, unless noted otherwise, and line intensity ratios are expressed in photon units.

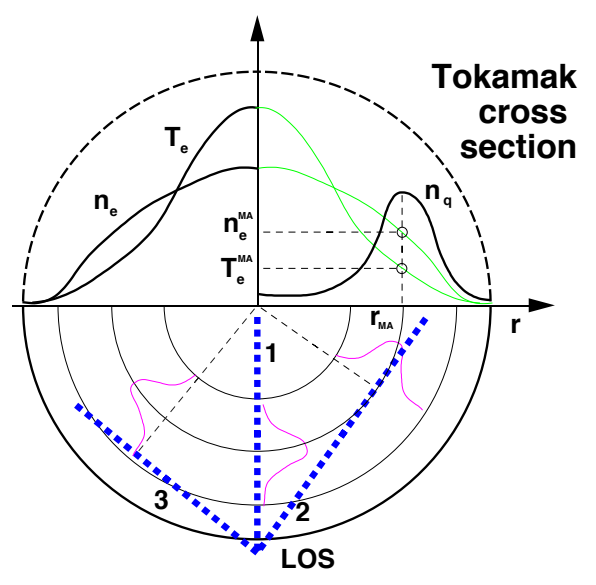

Fig. 2. Schematic view of temperature $\left(T_{\mathrm{e}}\right)$, density $\left(n_{\mathrm{e}}\right)$ and impurity density $\left(n_{q}\right)$ profiles (upper part of the drawing). The procedure of obtaining $T_{\mathrm{e}}$ and $n_{\mathrm{e}}$ at the maximum abundance (MA) location of each ion is shown. Lower part: bold dashed line indicates different lines of sight (LOS) of the spectrometer (1) - central, (2) - MA, (3) - plasma periphery

\section{Experiment and data analysis}

As mentioned, data from the experiments conducted at the TEXT and FTU tokamaks have been used in the present paper. The former experiment has been described by Lippmann et al. (1987), Finkenthal et al. (1986) and Finkenthal et al. (1987). The details of both experiments will be briefly mentioned here.

\subsection{Spectroscopic instrumentation}

The spectra were recorded by a 1 meter Rowland circle grazing incidence time-resolving spectrometer (GRITS) (Hodge et al. 1984). In both experiments, GRITS had a spectral resolution of $0.8 \AA$ (full width at half maximum (FWHM) of $0.7 \AA$ ). Temporal resolution (detector integration time, called a frame) was $5.4 \mathrm{~ms}$ in the TEXT experiment and $11 \mathrm{~ms}$ in the FTU experiment. A spectral range 60 to $80 \AA$ wide was covered by the detector during each tokamak discharge. The wavelength calibration was done using resonant lines of intrinsic and seeded tokamak impurities and is considered to be accurate to $0.2 \AA$. In both experiments, GRITS image intensified photodiode array detector was absolutely calibrated in the spectral range of $20-350 \AA$ using synchrotron radiation from the NIST SURF II electron storage ring. The uncertainty in the absolute brightness measurements was estimated to be $\sim 30 \%$, whereas relative brightness of two lines widely separated in wavelength was estimated to be $\leq 20 \%$. In the TEXT experiment, the edge plasma was monitored by a normal incidence time-resolved spectrometer (NITS) (Bell et al. 1981), covering the range between 300 and $2200 \AA$ A. At FTU, a SPRED (survey poor resolution extended domain) spectrometer (Fonk 1982) was used for measurements in the $200-1700 \AA$ range. 


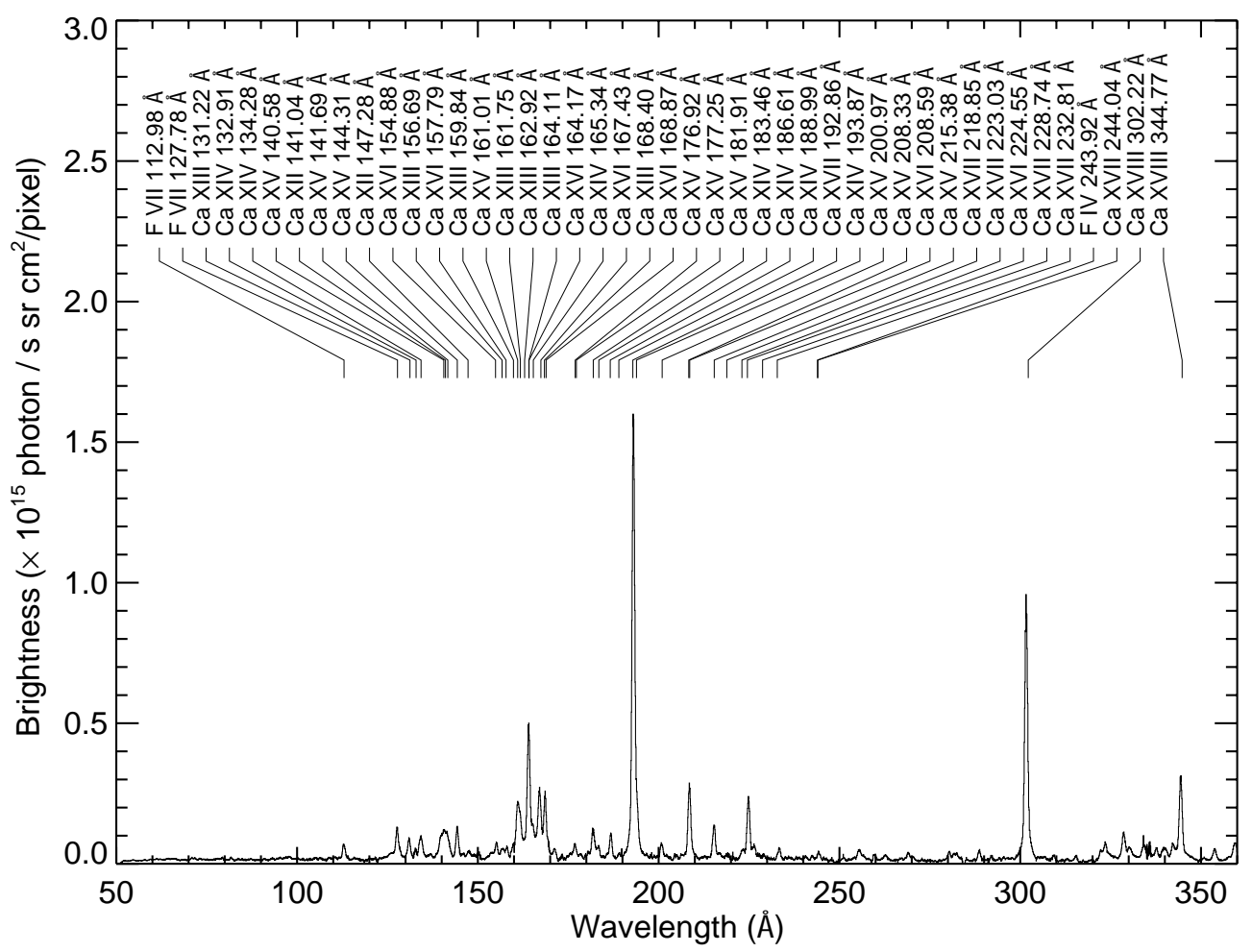

Fig. 3. Line-integrated, time-averaged XUV spectrum of highly ionized calcium in the TEXT tokamak. The spectrum was obtained using six reproduceable discharges

\subsection{Plasma diagnostics}

TEXT was a medium-size tokamak with a central electron temperature about $1 \mathrm{keV}$ and central electron density $\simeq 610^{13} \mathrm{~cm}^{-3}$ (Gentle 1981). The electron temperature was measured in the steady state by radially resolved Thompson scattering, and far infrared (FIR) interferometry was used for electron density diagnostics. The TEXT temperature and density profiles and other plasma parameters are given in Lippmann et al. (1987). FTU is a compact high magnetic field, high density tokamak (Andreani 1993). Typical time histories of its density and temperature profiles are shown in Fig. 1, as measured by FIR and electron cyclotron emission (ECE) interferometers, respectively. Thomson scattering measurements of $T_{\mathrm{e}}$ and $n_{\mathrm{e}}$ were also available. The accuracy of $T_{\mathrm{e}}$ measurements decreases with distance from the plasma center and is only good to $\pm 50 \mathrm{eV}$ in the plasma periphery. Calcium fluoride $\left(\mathrm{CaF}_{2}\right)$ was injected into the plasma in both experiments using a laser blow-off (LBO) technique (Terry et al. 1983). This method caused controlled perturbation to the plasma. The central electron temperature decrease was $14 \%$ at TEXT and $25 \%$ at FTU. The electron density increased by $\sim 18 \%$ at TEXT and $10 \%$ at FTU. The overall reproducibility of the plasma parameters and those of $\mathrm{CaF}_{2}$ injections was estimated to be within $10 \%$ in both experiments.

\subsection{Spectra interpretation}

Some of the recorded spectra are presented in Figs. 3 and 4. The TEXT spectrum was composed of six overlapping spectra obtained from reproduceable consequent discharges. Emission from intrinsic tokamak impurities (such as oxygen, carbon, iron, titanium) was integrated over several detector time frames preceding the $\mathrm{CaF}_{2}$ injection and subtracted from the injection spectrum. This procedure also helps to correct for the scattered light contribution to the recorded spectra. Line identifications were made using wavelength values compiled by Kelly (1987). Line intensities were obtained by piece-wise fitting of a multiGaussian function to the data using the wavelength from Kelly (1987). Analysis of the recorded spectra included: (i) interpretation of spectral line intensities of individual charge states and (ii) interpretation of full spectra. Lineintegrated intensities are characteristic of plasma conditions at the maximum abundance (MA) location of each ion (Fig. 2). Therefore, local plasma conditions $\left(T_{\mathrm{e}}\right.$ and $n_{\mathrm{e}}$ ) and emissivity distribution of each ion must be known. To achieve this, measurements were performed along different LOS over a number of reproduceable discharges in the TEXT tokamak. Line-integrated brightnesses of most intense spectral lines were Abel-inverted and species' radial distribution profiles were inferred (Fig. 3 in Lippmann et al. 1987). Relative line intensities of the considered $\Delta n=0$ transitions are practically temperature independent. Tables 2, 3 and 4 compare measured and calculated 


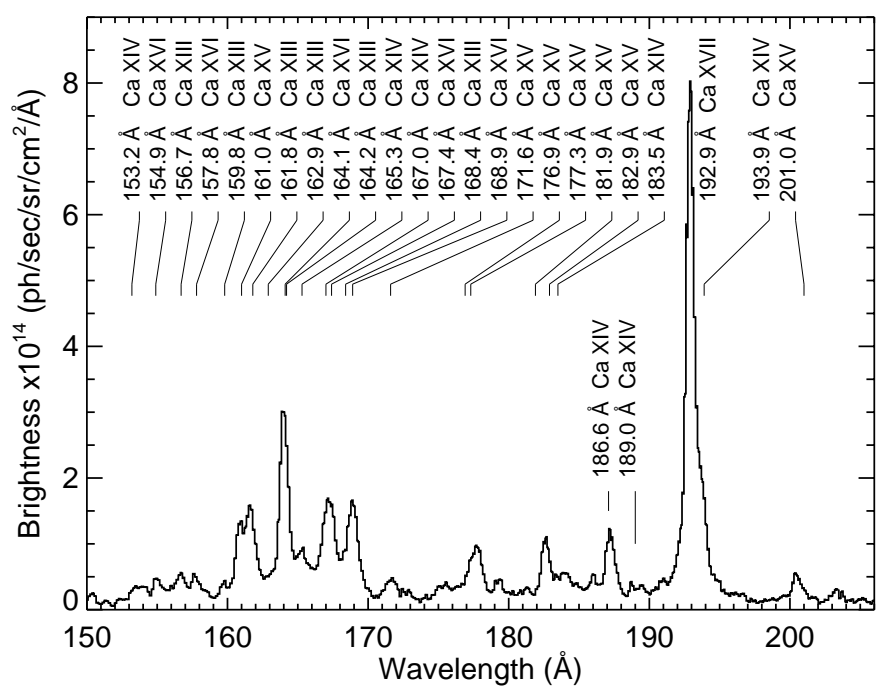

Fig. 4. LOS-integrated Ca spectrum, recorded at FTU

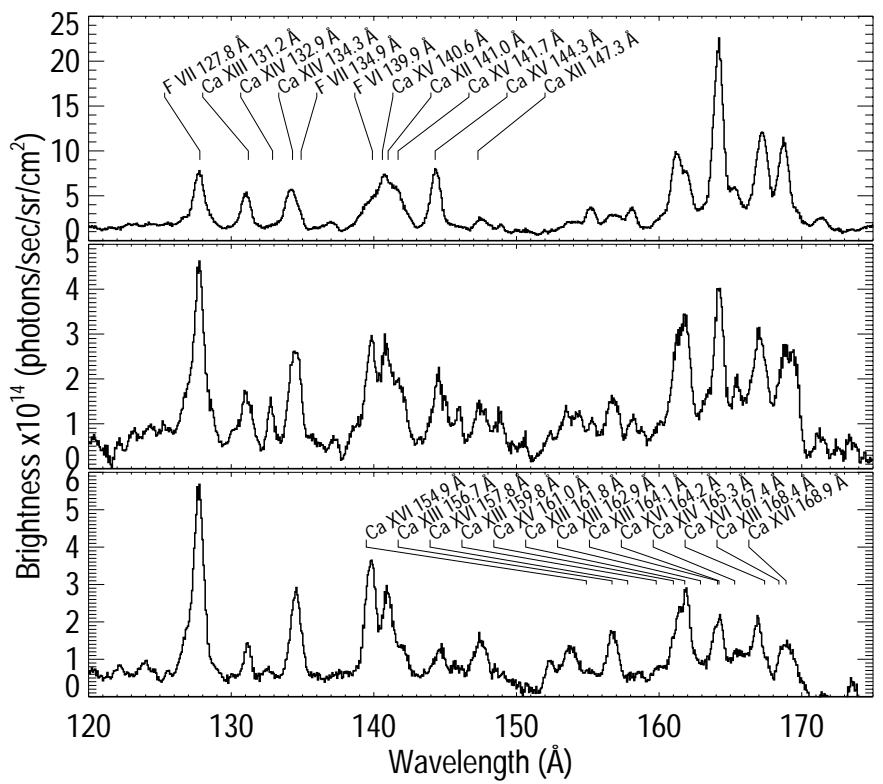

Fig. 5. TEXT spectra taken along three different lines of sight a) - through CaXvi MA location b) - through Ca XIV MA location, and c) - through Ca XII MA location

line intensities, normalized to the intensity of some resonant representative line of the same ion. The chosen normalization is fairly arbitrary, and meaningful comparisons are made on a case to case basis.

Spectral line blending presented problems in some cases. The blends can be classified as follows: calciumspecific blends (two or more close lines, emitted by calcium ions); blends due to the limited instrumental resolution; and blends, specific to the $\mathrm{CaF}_{2}$ injection. The spectral blend separation techniques used were similar to those described by Huang et al. (1987). Time behavior of LBO-injected impurities in a tokamak plasma is characterized by line brightness decay time. It is therefore possible to separate lines from different ionization stages
Table 1. Electron temperature and density at the maximum abundance location of Ca XVIII - Ca XII ions in TEXT and FTU tokamaks

\begin{tabular}{lccccc}
\hline & & \multicolumn{2}{c}{$k T_{\mathrm{e}}(\mathrm{eV})$} & \multicolumn{2}{c}{$n_{\mathrm{e}}\left(\times 10^{13} \mathrm{~cm}^{-3}\right)$} \\
\cline { 3 - 6 } Ion & I. P. $^{\mathrm{a}}(\mathrm{eV})$ & TEXT & FTU & TEXT & FTU \\
\hline CaXVIII & 1157 & $1000^{\mathrm{c}}$ & $650^{\mathrm{c}}$ & $5.4^{\mathrm{c}}$ & $3.0^{\mathrm{c}}$ \\
Ca XVII & 1087 & $800^{\mathrm{b}}$ & $550^{\mathrm{c}}$ & $4.0^{\mathrm{b}}$ & $2.8^{\mathrm{c}}$ \\
Ca XVI & 974 & $650^{\mathrm{b}}$ & $500^{\mathrm{c}}$ & $3.3^{\mathrm{b}}$ & $2.7^{\mathrm{c}}$ \\
Ca XV & 895 & $450^{\mathrm{b}}$ & $430^{\mathrm{c}}$ & $2.9^{\mathrm{b}}$ & $2.6^{\mathrm{c}}$ \\
Ca XIV & 818 & $350^{\mathrm{b}}$ & $360^{\mathrm{c}}$ & $2.4^{\mathrm{b}}$ & $2.4^{\mathrm{c}}$ \\
Ca XIII & 727 & $230^{\mathrm{b}}$ & $240^{\mathrm{c}}$ & $1.8^{\mathrm{b}}$ & $2.2^{\mathrm{c}}$ \\
Ca XII & 657 & $130^{\mathrm{b}}$ & $160^{\mathrm{c}}$ & $1.2^{\mathrm{b}}$ & $2.0^{\mathrm{c}}$ \\
\hline
\end{tabular}

${ }^{a}$ Ionization potential, from Kelly (1987).

${ }^{\mathrm{b}}$ From measured ion emissivity profiles and measured $T_{\mathrm{e}}$ and $n_{\mathrm{e}}$ profiles as described in Sect. 2 .

${ }^{c}$ Calculated as described in Sect. 2.

by comparing time histories of adjacent detector pixels. In the cases where the measured lines originate from the same upper level, branching ratios of the corresponding transitions were used to estimate blended line intensities. Also, spectra recorded at different spatial locations (and therefore at different temperatures), were used in the analysis. For example, Ca XII lines $\lambda 141.0$ and $\lambda 147.3$ are strongly blended with the brighter lines of $\mathrm{CaXV}$ and CaXIV on a spectrum recorded along the near-central line of sight (LOS) (Fig. 5). The spectrum with the LOS passing through the CaXII emissivity peak was used to derive the $\lambda 141.1$ and $\lambda 147.3$ line brightnesses. The radial position, according to the measured temperature profile, corresponds to $\sim 130 \mathrm{eV}$. In some cases (such as the CaXVIII lines recorded at TEXT, and the FTU spectra (Fig. 4)), spatial measurements were not performed. The predicted ground state density distributions were used to estimate the ion MA temperature and density, as described below.

In a tokamak discharge, ground state densities of impurity ions are constrained by radial temperature and density gradients and the radial particle transport. The tokamak plasma is optically thin, and the main inter-species processes are recombination (radiative, dielectronic) and ionization (direct, autoionization). In a typical ohmically heated tokamak plasma after an LBO impurity injection, ion species evolve toward a steady state equilibrium. This was confirmed in many experiments: for example, Horton \& Rowan (1994) extensively studied transport phenomena in the TEXT tokamak, using Sc and Ti, LBO-injected into plasma. However, ion maximum abundance temperatures differ from the coronal ionization-recombination equilibrium case due to inward radial plasma transport. The ion fractional abundance peaks are shifted toward higher temperatures (Table 1). Time evolution of the ground level density $n_{q}$ 
of injected impurity ion species $q$ can be obtained by solving a set of differential equations:

$$
\begin{aligned}
\frac{\mathrm{d} n_{q}}{\mathrm{~d} t}=-\frac{1}{r} \frac{\partial}{\partial r} r \Gamma_{q}- & \frac{n_{q}}{\tau_{q}}+S_{q}+ \\
& I_{q-1} n_{q-1}-\left(I_{q}+R_{q}\right) n_{q}+R_{q+1} n_{q+1},
\end{aligned}
$$

where $q$ is ion charge $\left(0 \leq q \leq 20\right.$ for calcium), $\Gamma_{q}$ is radial particle flux, $S_{q}$ and $-\frac{n_{q}}{\tau_{q}}$ are impurity source and sink terms, respectively, and $I_{q}, R_{q}$ are total ionization and recombination rates. To obtain fractional abundances, the set of equations was solved by the transport code MIST (Hulse 1983). Measured $T_{\mathrm{e}}$ and $n_{\mathrm{e}}$ profiles were used as input for this calculation. Transport parameters, such as diffusion and convection coefficients, which enter the equations through the particle flux quantity $\Gamma$, were adopted from the dedicated transport experiments (Horton \& Rowan 1994).

\section{Atomic data and collisional-radiative model}

\subsection{Details of computations}

The atomic data for $\mathrm{CR}$ modeling was generated by the HULLAC suite of computer codes. HULLAC computes the ab initio intermediate coupled wave functions, level energies and transition probabilities, using the fully relativistic parametric potential code RELAC (Klapisch 1971; Klapisch et al. 1977). All double excited states with energies less than or equal to the energy of the highest included resonant level were also included in the model for each ion. The electron collision strengths were calculated in distorted wave (DW) approximation and averaged over Maxwellian distribution to produce collisional excitation rates by the CROSS code (Bar-Shalom et al. 1988). Effects of proton-ion collisions were not included in our model. Proton collisions can change populations of closely spaced fine structure levels, such as CaXVI - CaXIII ground levels. Stratton et al. (1985) demonstrated that for the $\mathrm{Ti}, \mathrm{Cr}, \mathrm{Fe}$ and $\mathrm{Ni}$ ions this effect is weak, with the exception of carbon-like ions. Since electron impact excitation is the main source of excited level population at the typical tokamak temperature and density, the accuracy of the electron excitation rate coefficients is critical for calculating accurate spectral line intensities. This especially concerns the ions which have metastable levels with relatively large populations. The DW approximation does not take into account scattering resonances and coupling between scattering channels. Finkenthal et al. (1987) pointed out the importance of these effects for CR modeling of lower $\mathrm{Z}$ elements. In the described tokamak experiments electron energies are typically much greater than collisional excitation thresholds of the XUV lines, and the DW approximation, therefore, proves adequate. There has been a number of theoretical studies which compare R-matrix and
DW calculated electron collision strengths for CaXVIII - CaXIII ions (for example, Huang et al. 1987; Bhatia et al. 1986; Dufton et al. 1983; Zhang \& Pradhan 1994; Bhatia \& Doschek 1993; Aggarwal 1992; Baliyan \& Bhatia 1994). The collision strengths, calculated by the two methods, agree to better than $30 \%$ for the temperature range considered $(100-1000 \mathrm{eV})$. Therefore, the DW excitation rate coefficients, used in this work, are of adequate accuracy.

Using the ab initio level energies, transition probabilities and collisional excitation rate coefficients generated by HULLAC, quasi-steady state (QSS) level population calculations were performed for the temperatures and densities of interest. All E1, M1 and M2 radiative transitions and all collisional excitation and deexcitation transitions were included. The quadrupole (E2) radiative transitions were found to be negligible for the L-shell calcium ions and were not included in the models. For the extended detailed non-LTE calculations, which involved the adjacent ion species, ionization and recombination rates were generated as follows: ionization rates, including inner-shell, were calculated according to the Lotz formula (Lotz 1968, 1970) using the ab initio level energies. Autoionization probabilities were calculated by RELAC in the DW approximation. Recombination rates were calculated based on detailed balance principle. In particular, dielectronic recombination was taken into account by calculating radiationless capture rates from the ab initio autoionization rates.

Atomic rates from CHIANTI database were also used for CR calculations of line intensities. To the extent of our measurements, a large set of CHIANTI calcium data has been benchmarked in the present work. CHIANTI includes the best available electron impact excitation and radiative decay rates for $\mathrm{E} 1$ and $\mathrm{M} 1$ transitions of $n=2$ configurations of CaXV, Ca XIV, Ca XII, $n=2,3$ configurations of CaXVII, CaXVI, CaXIII, and $n=2,3,4,5$ configurations of CaXVIII (Dere et al. 1997; Landi et al. 1999).

\subsection{Model ions}

Two models were generated for each ion: a basic model and an extended model. The basic models included a reduced number of levels and basic atomic processes (collisional excitation and de-excitation and radiative decay), and were found to be sufficient for most cases. The extended models were generated for particular test cases and therefore included greater number of levels and additional atomic processes (such as K-shell excitation, autoionization, collisional ionization from metastable and excited levels). The effect of radiative cascades on the populations of $n=2$ levels is found to be $\leq 15 \%$ for the transitions from $n=3$ levels and $\leq 5 \%$ from $n=4,5$ levels. For the range of plasma parameters of both experiments, we have checked and concluded that inner-shell 
Table 2. Observed and calculated relative line intensities of Ca XVIII, Ca XVII, Ca XVI, Ca XV in TEXT tokamak

\begin{tabular}{|c|c|c|c|c|c|c|c|c|}
\hline \multirow[b]{3}{*}{ Ion } & \multirow{2}{*}{\multicolumn{2}{|c|}{ Lower level }} & \multirow{2}{*}{\multicolumn{2}{|c|}{ Upper level }} & \multirow[b]{3}{*}{$\lambda(\AA)^{\mathrm{c}}$} & \multicolumn{3}{|c|}{ Relative Intensity $^{\mathrm{a}}$} \\
\hline & & & & & & \multirow[t]{2}{*}{ Measured } & \multicolumn{2}{|c|}{ Calculated $^{\mathrm{d}}$} \\
\hline & Term & Label $^{b}$ & Term & Label $^{\text {b }}$ & & & HULLAC & CHIANTI \\
\hline \multirow[t]{2}{*}{ Ca XviII } & $2 \mathrm{~s}^{2} \mathrm{~S}_{1 / 2}$ & 1 & $2 \mathrm{p}^{2} \mathrm{P}_{3 / 2}$ & 3 & 302.2 & 100 & 100.0 & 100.0 \\
\hline & $2 \mathrm{~s}^{2} \mathrm{~S}_{1 / 2}$ & 1 & $2 \mathrm{p}^{2} \mathrm{P}_{1 / 2}$ & 2 & 344.8 & 32 & 51.5 & 51.1 \\
\hline \multirow[t]{7}{*}{ Ca XVII } & $2 \mathrm{~s}^{2}{ }^{1} \mathrm{~S}_{0}$ & 1 & $2 \mathrm{~s} 2 \mathrm{p}^{1} \mathrm{P}_{1}$ & 5 & 192.9 & 100 & 100.0 & 100.0 \\
\hline & $2 \mathrm{~s} 2 \mathrm{p}^{3} \mathrm{P}_{1}$ & 3 & $2 \mathrm{p}^{2}{ }^{3} \mathrm{P}_{2}$ & 8 & 218.8 & 1.0 & 0.93 & 0.85 \\
\hline & $2 \mathrm{~s} 2 \mathrm{p}^{3} \mathrm{P}_{0}$ & 2 & $2 \mathrm{p}^{23} \mathrm{P}_{1}$ & 7 & 223.0 & 1.1 & 0.75 & 0.73 \\
\hline & $2 \mathrm{~s} 2 \mathrm{p}^{3} \mathrm{P}_{1}$ & 3 & $2 \mathrm{p}^{2}{ }^{3} \mathrm{P}_{1}$ & 7 & 228.7 & 1.1 & 0.51 & 0.50 \\
\hline & $2 \mathrm{~s} 2 \mathrm{p}^{3} \mathrm{P}_{2}$ & 4 & $2 \mathrm{p}^{2}{ }^{3} \mathrm{P}_{2}$ & 8 & 232.8 & 2.0 & 2.15 & 1.95 \\
\hline & $2 \mathrm{~s} 2 \mathrm{p}^{3} \mathrm{P}_{2}$ & 4 & $2 \mathrm{p}^{23} \mathrm{P}_{1}$ & 7 & 244.0 & 1.4 & 0.70 & 0.69 \\
\hline & $2 \mathrm{~s}^{2}{ }^{1} \mathrm{~S}_{0}$ & 1 & $2 \mathrm{~s} 2 \mathrm{p}^{3} \mathrm{P}_{1}$ & 3 & 371.0 & $\cdots$ & 2.80 & 2.92 \\
\hline \multirow[t]{7}{*}{ Ca XVI } & $2 \mathrm{~s}^{2} 2 \mathrm{p}^{2} \mathrm{P}_{1 / 2}$ & 1 & $2 \mathrm{~s} 2 \mathrm{p}^{2}{ }^{2} \mathrm{P}_{3 / 2}$ & 10 & 154.9 & 18 & 17.8 & 17.7 \\
\hline & $2 \mathrm{~s}^{2} 2 \mathrm{p}^{2} \mathrm{P}_{1 / 2}$ & 1 & $2 \mathrm{~s} 2 \mathrm{p}^{2}{ }^{2} \mathrm{P}_{1 / 2}$ & 9 & 157.8 & 10 & 17.2 & 13.3 \\
\hline & $2 \mathrm{~s}^{2} 2 \mathrm{p}^{2} \mathrm{P}_{3 / 2}$ & 2 & $2 \mathrm{~s} 2 \mathrm{p}^{2}{ }^{2} \mathrm{P}_{3 / 2}$ & 10 & 164.2 & 100 & 100.0 & 100.0 \\
\hline & $2 \mathrm{~s}^{2} 2 \mathrm{p}^{2} \mathrm{P}_{3 / 2}$ & 2 & $2 \mathrm{~s} 2 \mathrm{p}^{2}{ }^{2} \mathrm{P}_{1 / 2}$ & 9 & 167.4 & 34 & 39.8 & 47.9 \\
\hline & $2 \mathrm{~s}^{2} 2 \mathrm{p}^{2} \mathrm{P}_{1 / 2}$ & 1 & $2 \mathrm{~s} 2 \mathrm{p}^{2}{ }^{2} \mathrm{~S}_{1 / 2}$ & 8 & 168.9 & 26 & 48.3 & 67.4 \\
\hline & $2 \mathrm{~s}^{2} 2 \mathrm{p}^{2} \mathrm{P}_{1 / 2}$ & 1 & $2 \mathrm{~s} 2 \mathrm{p}^{2}{ }^{2} \mathrm{D}_{3 / 2}$ & 6 & 208.6 & 32 & 54.8 & 67.3 \\
\hline & $2 \mathrm{~s}^{2} 2 \mathrm{p}^{2} \mathrm{P}_{3 / 2}$ & 2 & $2 \mathrm{~s} 2 \mathrm{p}^{2}{ }^{2} \mathrm{D}_{5 / 2}$ & 7 & 224.6 & 40 & 71.3 & 73.5 \\
\hline \multirow[t]{14}{*}{$\mathrm{Caxv}$} & $2 \mathrm{~s}^{2} 2 \mathrm{p}^{2}{ }^{3} \mathrm{P}_{0}$ & 1 & $2 \mathrm{~s} 2 \mathrm{p}^{3}{ }^{3} \mathrm{~S}_{1}$ & 13 & 137.2 & 20 & 15.8 & 15.6 \\
\hline & $2 \mathrm{~s}^{2} 2 \mathrm{p}^{2}{ }^{3} \mathrm{P}_{1}$ & 2 & $2 \mathrm{~s} 2 \mathrm{p}^{3}{ }^{3} \mathrm{~S}_{1}$ & 13 & 140.6 & 42 & 45.7 & 45.7 \\
\hline & $2 \mathrm{~s}^{2} 2 \mathrm{p}^{2}{ }^{1} \mathrm{D}_{2}$ & 4 & $2 \mathrm{~s} 2 \mathrm{p}^{3}{ }^{1} \mathrm{P}_{1}$ & 15 & 141.7 & 51 & 47.9 & 48.8 \\
\hline & $2 \mathrm{~s}^{2} 2 \mathrm{p}^{2}{ }^{3} \mathrm{P}_{2}$ & 3 & $2 \mathrm{~s} 2 \mathrm{p}^{3}{ }^{1} \mathrm{D}_{2}$ & 14 & 144.3 & 107 & 85.5 & 86.2 \\
\hline & $2 \mathrm{~s}^{2} 2 \mathrm{p}^{2}{ }^{1} \mathrm{D}_{2}$ & 4 & $2 \mathrm{~s} 2 \mathrm{p}^{3}{ }^{1} \mathrm{D}_{2}$ & 14 & 161.0 & 100 & 100.0 & 100.0 \\
\hline & $2 \mathrm{~s}^{2} 2 \mathrm{p}^{2}{ }^{3} \mathrm{P}_{0}$ & 1 & $2 \mathrm{~s} 2 \mathrm{p}^{3}{ }^{3} \mathrm{P}_{1}$ & 11 & 171.6 & 35 & 18.9 & 17.9 \\
\hline & $2 \mathrm{~s}^{2} 2 \mathrm{p}^{2}{ }^{3} \mathrm{P}_{1}$ & 2 & $2 \mathrm{~s} 2 \mathrm{p}^{3}{ }^{3} \mathrm{P}_{2}$ & 12 & 176.0 & 8 & 12.2 & 11.8 \\
\hline & $2 \mathrm{~s}^{2} 2 \mathrm{p}^{2}{ }^{3} \mathrm{P}_{1}$ & 2 & $2 \mathrm{~s} 2 \mathrm{p}^{3}{ }^{3} \mathrm{P}_{1}$ & 11 & 176.9 & 47 & 31.3 & 29.8 \\
\hline & $2 \mathrm{~s}^{2} 2 \mathrm{p}^{2}{ }^{3} \mathrm{P}_{1}$ & 2 & $2 \mathrm{~s} 2 \mathrm{p}^{3}{ }^{3} \mathrm{P}_{0}$ & 10 & 177.3 & 20 & 23.5 & 23.8 \\
\hline & $2 \mathrm{~s}^{2} 2 \mathrm{p}^{2}{ }^{3} \mathrm{P}_{2}$ & 3 & $2 \mathrm{~s} 2 \mathrm{p}^{3}{ }^{3} \mathrm{P}_{2}$ & 12 & 181.9 & 60 & 95.9 & 94.2 \\
\hline & $2 \mathrm{~s}^{2} 2 \mathrm{p}^{2}{ }^{3} \mathrm{P}_{2}$ & 3 & $2 \mathrm{~s} 2 \mathrm{p}^{3}{ }^{3} \mathrm{P}_{1}$ & 11 & 182.9 & 15 & 19.8 & 18.0 \\
\hline & $2 \mathrm{~s}^{2} 2 \mathrm{p}^{2}{ }^{3} \mathrm{P}_{0}$ & 1 & $2 \mathrm{~s} 2 \mathrm{p}^{3}{ }^{3} \mathrm{D}_{1}$ & 8 & 201.0 & 29 & 41.2 & 38.8 \\
\hline & $2 \mathrm{~s}^{2} 2 \mathrm{p}^{2}{ }^{3} \mathrm{P}_{1}$ & 2 & $2 \mathrm{~s} 2 \mathrm{p}^{3}{ }^{3} \mathrm{D}_{2}$ & 7 & 208.7 & 82 & 82.3 & 77.9 \\
\hline & $2 \mathrm{~s}^{2} 2 \mathrm{p}^{2}{ }^{3} \mathrm{P}_{2}$ & 3 & $2 \mathrm{~s} 2 \mathrm{p}^{3}{ }^{3} \mathrm{D}_{3}$ & 9 & 215.4 & 95 & 106.0 & 101.4 \\
\hline
\end{tabular}

${ }^{a}$ Line intensity, relative to some representative line in each isosequence.

${ }^{\mathrm{b}}$ Labels refer to Fig. 7 .

${ }^{\mathrm{c}}$ From Kelly (1987).

${ }^{\mathrm{d}}$ For $T_{\mathrm{e}}$ and $n_{\mathrm{e}}$ values, corresponding to the measured MA location of the ion.

ionization does not change the relative level populations significantly $(\leq 15 \%)$, which confirms applicability of the steady state equilibrium. Total ionization rates from the $n=3$, 4 resonant levels were found to be a factor of $10^{1}-10^{2}$ less than total radiative decay rates from these levels.

CaXVIII - The basic model for lithium-like calcium includes 67 energetically lowest levels from the configurations $1 \mathrm{~s}^{2} 2 l(l=\mathrm{s}, \mathrm{p}), 1 \mathrm{~s}^{2} 3 l(l=\mathrm{s}, \mathrm{p}, \mathrm{d}), 1 \mathrm{~s}^{2} 4 l$ $(l=\mathrm{s}, \mathrm{p}, \mathrm{d}, \mathrm{f}), 1 \mathrm{~s} 2 \mathrm{~s} 2 l(l=\mathrm{s}, \mathrm{p}), 1 \mathrm{~s} 2 \mathrm{~s} 3 l(l=\mathrm{s}, \mathrm{p}, \mathrm{f})$, 1s $2 \mathrm{~s} 4 l(l=\mathrm{s}, \mathrm{p}, \mathrm{d}, \mathrm{f})$. The extended model includes levels up to $n=5$, including configurations of the type $1 \mathrm{~s} 2 \mathrm{~s} n l$. A He-like calcium model with all levels up to $n=4$ was also used.

Ca XVII - Our basic model for beryllium-like calcium ion included 125 levels of the configurations $1 \mathrm{~s}^{2} 2 \mathrm{~s}^{2}, 1 \mathrm{~s}^{2} 2 \mathrm{~s} n l$, and $1 \mathrm{~s}^{2} 2 l^{\prime} n l^{\prime \prime}$, where $n=2,3,4$. The extended model comprises configurations of the type $1 \mathrm{~s}^{2} \mathrm{~s}^{2} n l(n=2,3,4)$ and $1 \mathrm{~s} 2 \mathrm{~s} 2 \mathrm{p} n l(n=2,3,4)$.

CaXVI - The configurations $2 \mathrm{~s}^{2} n l, 2 \mathrm{~s} 2 \mathrm{p} n l(n=2, l=\mathrm{s}$, $\mathrm{p} ; n=3, l=\mathrm{s}, \mathrm{p}, \mathrm{d} ; n=4 ; l=\mathrm{s}, \mathrm{p}, \mathrm{d}, \mathrm{f})$ and $2 \mathrm{~s} 2 \mathrm{p}^{3}$ are included in the basic model of 147 levels. The extended model, in addition to the configurations listed above, includes the $5 l(l=\mathrm{s}, \mathrm{p}, \mathrm{d}, \mathrm{f})$ and the $1 \mathrm{~s} 2 \mathrm{~s}^{2} 2 \mathrm{p} n l(n=2$, $l=p ; n=3, l=\mathrm{s}, \mathrm{p}, \mathrm{d}$, and $n=4, l=\mathrm{s}, \mathrm{p})$.

CaXV - The basic model ion includes 377 levels of 
Table 3. Observed and calculated relative line intensities of Ca XIV, CaXIII, Ca XII in TEXT tokamak

\begin{tabular}{|c|c|c|c|c|c|c|c|c|}
\hline \multirow[b]{3}{*}{ Ion } & \multirow{2}{*}{\multicolumn{2}{|c|}{ Lower level }} & \multirow{2}{*}{\multicolumn{2}{|c|}{ Upper level }} & \multirow[b]{3}{*}{$\lambda(\AA)^{\mathrm{c}}$} & \multicolumn{3}{|c|}{ Relative Intensity $^{\mathrm{a}}$} \\
\hline & & & & & & \multirow[t]{2}{*}{ Measured } & \multicolumn{2}{|c|}{ Calculated $^{\mathrm{d}}$} \\
\hline & Term & $\mathrm{Label}^{\mathrm{b}}$ & Term & Label $^{b}$ & & & HULLAC & CHIANTI \\
\hline \multirow[t]{11}{*}{ Ca XIV } & $2 \mathrm{~s}^{2} 2 \mathrm{p}^{32} \mathrm{D}_{3 / 2}$ & 2 & $2 \mathrm{~s} 2 \mathrm{p}^{42} \mathrm{P}_{1 / 2}$ & 13 & 128.2 & 15 & 12.0 & 17.0 \\
\hline & $2 \mathrm{~s}^{2} 2 \mathrm{p}^{32} \mathrm{D}_{3 / 2}$ & 2 & $2 \mathrm{~s} 2 \mathrm{p}^{42} \mathrm{P}_{3 / 2}$ & 12 & 132.9 & 12 & 9.1 & 13.5 \\
\hline & $2 \mathrm{~s}^{2} 2 \mathrm{p}^{32} \mathrm{D}_{5 / 2}$ & 3 & $2 \mathrm{~s} 2 \mathrm{p}^{42} \mathrm{P}_{3 / 2}$ & 12 & 134.3 & 34 & 46.1 & 67.5 \\
\hline & $2 \mathrm{~s}^{2} 2 \mathrm{p}^{32} \mathrm{P}_{3 / 2}$ & 5 & $2 \mathrm{~s} 2 \mathrm{p}^{42} \mathrm{P}_{1 / 2}$ & 13 & 142.4 & 10 & 12.5 & 19.7 \\
\hline & $2 \mathrm{~s}^{2} 2 \mathrm{p}^{32} \mathrm{P}_{1 / 2}$ & 4 & $2 \mathrm{~s} 2 \mathrm{p}^{42} \mathrm{~S}_{1 / 2}$ & 11 & 153.2 & 6 & 9.0 & 13.6 \\
\hline & $2 \mathrm{~s}^{2} 2 \mathrm{p}^{32} \mathrm{D}_{3 / 2}$ & 2 & $2 \mathrm{~s} 2 \mathrm{p}^{42} \mathrm{D}_{3 / 2}$ & 9 & 165.3 & 39 & 38.0 & 53.3 \\
\hline & $2 \mathrm{~s}^{2} 2 \mathrm{p}^{32} \mathrm{D}_{5 / 2}$ & 3 & $2 \mathrm{~s} 2 \mathrm{p}^{42} \mathrm{D}_{5 / 2}$ & 10 & 167.0 & 60 & 51.6 & 67.7 \\
\hline & $2 \mathrm{~s}^{2} 2 \mathrm{p}^{3}{ }^{4} \mathrm{~S}_{3 / 2}$ & 1 & $2 \mathrm{~s} 2 \mathrm{p}^{4{ }^{4}} \mathrm{P}_{1 / 2}$ & 8 & 183.5 & 26 & 32.7 & 33.9 \\
\hline & $2 \mathrm{~s}^{2} 2 \mathrm{p}^{3{ }^{4}} \mathrm{~S}_{3 / 2}$ & 1 & $2 \mathrm{~s} 2 \mathrm{p}^{44} \mathrm{P}_{3 / 2}$ & 7 & 186.6 & 43 & 65.4 & 66.0 \\
\hline & $2 \mathrm{~s}^{2} 2 \mathrm{p}^{32} \mathrm{P}_{3 / 2}$ & 5 & $2 \mathrm{~s} 2 \mathrm{p}^{42} \mathrm{D}_{5 / 2}$ & 10 & 189.0 & 10 & 10.3 & 14.1 \\
\hline & $2 \mathrm{~s}^{2} 2 \mathrm{p}^{3}{ }^{4} \mathrm{~S}_{3 / 2}$ & 1 & $2 \mathrm{~s} 2 \mathrm{p}^{4}{ }^{4} \mathrm{P}_{5 / 2}$ & 6 & 193.9 & 100 & 100.0 & 100.0 \\
\hline \multirow[t]{7}{*}{ Ca XIII } & $2 \mathrm{~s}^{2} 2 \mathrm{p}^{4}{ }^{1} \mathrm{D}_{2}$ & 4 & $2 \mathrm{~s} 2 \mathrm{p}^{5}{ }^{1} \mathrm{P}_{1}$ & 9 & 131.2 & 69 & 63.2 & 68.7 \\
\hline & $2 \mathrm{~s}^{2} 2 \mathrm{p}^{4}{ }^{3} \mathrm{P}_{2}$ & 1 & $2 \mathrm{~s} 2 \mathrm{p}^{5}{ }^{3} \mathrm{P}_{1}$ & 7 & 156.7 & 48 & 34.3 & 34.0 \\
\hline & $2 \mathrm{~s}^{2} 2 \mathrm{p}^{4}{ }^{3} \mathrm{P}_{1}$ & 2 & $2 \mathrm{~s} 2 \mathrm{p}^{5}{ }^{3} \mathrm{P}_{0}$ & 8 & 159.8 & 20 & 23.7 & 24.4 \\
\hline & $2 \mathrm{~s}^{2} 2 \mathrm{p}^{4}{ }^{3} \mathrm{P}_{2}$ & 1 & $2 \mathrm{~s} 2 \mathrm{p}^{5}{ }^{3} \mathrm{P}_{2}$ & 6 & 161.7 & 100 & 100.0 & 100.0 \\
\hline & $2 \mathrm{~s}^{2} 2 \mathrm{p}^{4}{ }^{3} \mathrm{P}_{1}$ & 2 & $2 \mathrm{~s} 2 \mathrm{p}^{5}{ }^{3} \mathrm{P}_{1}$ & 7 & 162.9 & 18 & 18.0 & 18.0 \\
\hline & $2 \mathrm{~s}^{2} 2 \mathrm{p}^{4}{ }^{3} \mathrm{P}_{0}$ & 3 & $2 \mathrm{~s} 2 \mathrm{p}^{5}{ }^{3} \mathrm{P}_{0}$ & 8 & 164.1 & 35 & 22.9 & 22.9 \\
\hline & $2 \mathrm{~s}^{2} 2 \mathrm{p}^{4}{ }^{3} \mathrm{P}_{1}$ & 2 & $2 \mathrm{~s} 2 \mathrm{p}^{5}{ }^{3} \mathrm{P}_{2}$ & 6 & 168.4 & 24 & 30.5 & 30.5 \\
\hline \multirow[t]{2}{*}{ Ca XII } & $2 \mathrm{~s}^{2} 2 \mathrm{p}^{5}{ }^{2} \mathrm{P}_{3 / 2}$ & 1 & $2 \mathrm{~s} 2 \mathrm{p}^{6}{ }^{2} \mathrm{~S}_{1 / 2}$ & 3 & 141.0 & 100.0 & 100.0 & 100.0 \\
\hline & $2 \mathrm{~s}^{2} 2 \mathrm{p}^{52} \mathrm{P}_{1 / 2}$ & 2 & $2 \mathrm{~s} 2 \mathrm{p}^{6}{ }^{2} \mathrm{~S}_{1 / 2}$ & 3 & 147.3 & 40 & 43.6 & 43.1 \\
\hline
\end{tabular}

${ }^{\mathrm{a}}$ Line intensity, relative to some representative line in each isosequence.

${ }^{\mathrm{b}}$ Labels refer to Fig. 7 .

${ }^{\mathrm{c}}$ From Kelly (1987).

${ }^{\mathrm{d}}$ For $T_{\mathrm{e}}$ and $n_{\mathrm{e}}$ values, corresponding to the measured MA location of the ion.

the configurations $2 \mathrm{~s}^{2} 2 \mathrm{p}^{2}, \quad 2 \mathrm{~s}^{2} 2 \mathrm{p} n l \quad 2 \mathrm{~s} 2 \mathrm{p}^{2} n l, \quad 2 \mathrm{~s}^{2} n l^{2}$ and $1 \mathrm{~s}^{2} 2 \mathrm{p}^{4}(n=2, l=\mathrm{s}, \mathrm{p} ; n=3, l=\mathrm{s}, \mathrm{p}, \mathrm{d}$; $n=4 ; l=\mathrm{s}, \mathrm{p}, \mathrm{d}, \mathrm{f})$. The extended model ion comprised a total of 1056 levels: the configurations mentioned above and the configurations $1 \mathrm{~s}^{2} 2 \mathrm{~s}^{2} 2 \mathrm{p} 5 l$ $(l=\mathrm{s}, \mathrm{p}, \mathrm{d}, \mathrm{f}), 1 \mathrm{~s}^{2} 2 \mathrm{~s}^{1} 2 \mathrm{p}^{2} 5 l(l=\mathrm{s}, \mathrm{p}, \mathrm{d}, \mathrm{f})$, and $1 \mathrm{~s} 2 \mathrm{~s}^{2} 2 \mathrm{p}^{2} n l(n=2, l=\mathrm{p} ; n=3, l=\mathrm{s}, \mathrm{p}, \mathrm{d} ; n=4,5, l$ $=\mathrm{s}, \mathrm{p}, \mathrm{d}, \mathrm{f})$.

CaXIV - We use the configurations $2 \mathrm{~s}^{2} 2 \mathrm{p}^{2} n l, 2 \mathrm{~s} 2 \mathrm{p}^{3} n l$ and $2 \mathrm{~s}^{2} n l n^{\prime} l^{\prime}$, where $n=2, l=\mathrm{s}, \mathrm{p} ; n=3, l=\mathrm{s}, \mathrm{p}, \mathrm{d} ; n=$ $4, l=\mathrm{s}, \mathrm{p}, \mathrm{d}$, f and $n^{\prime}=3, l^{\prime}=\mathrm{s}, \mathrm{p}, \mathrm{d}$. The total number of levels in the basic model is 546 .

Ca XIII - The model ion contains 542 levels of the following configurations: $2 \mathrm{~s}^{2} 2 \mathrm{p}^{4}, 2 \mathrm{~s}^{2} 2 \mathrm{p}^{3} n l(n=3 ; l=\mathrm{s}, \mathrm{p}, \mathrm{d}$ and $n=4 ; l=\mathrm{s}, \mathrm{p}, \mathrm{d}, \mathrm{f}), 2 \mathrm{~s} 2 \mathrm{p}^{4} n l(n=3 ; l=\mathrm{s}, \mathrm{p}, \mathrm{d}$ and $n$ $=4 ; l=\mathrm{s}, \mathrm{p}, \mathrm{d}, \mathrm{f}), 2 \mathrm{~s} 2 \mathrm{p}^{6}, 2 \mathrm{~s}^{2} 2 \mathrm{p}^{2} n l n^{\prime} l^{\prime}(n=3 ; l=\mathrm{s}, \mathrm{p}$ and $\left.n^{\prime}=3 ; l=\mathrm{s}, \mathrm{p}\right)$ and $2 \mathrm{~s}^{2} 3 \mathrm{~s}^{2} 3 \mathrm{p}^{2}$.

CaXII - The 267 levels of the configurations $2 \mathrm{~s}^{2} 2 \mathrm{p}^{5}$, $2 \mathrm{~s}^{2} 2 \mathrm{p}^{4} n l(n=3 ; l=\mathrm{s}, \mathrm{p}, \mathrm{d}$ and $n=4 ; l=\mathrm{s}, \mathrm{p}, \mathrm{d}, \mathrm{f}), 2 \mathrm{~s}$ $2 \mathrm{p}^{6}$ and $2 \mathrm{~s} 2 \mathrm{p}^{5} n l(n=3 ; l=\mathrm{s}, \mathrm{p}, \mathrm{d}$ and $n=4 ; l=\mathrm{s}, \mathrm{p}$, $\mathrm{d}, \mathrm{f})$ are used in the basic model ion.

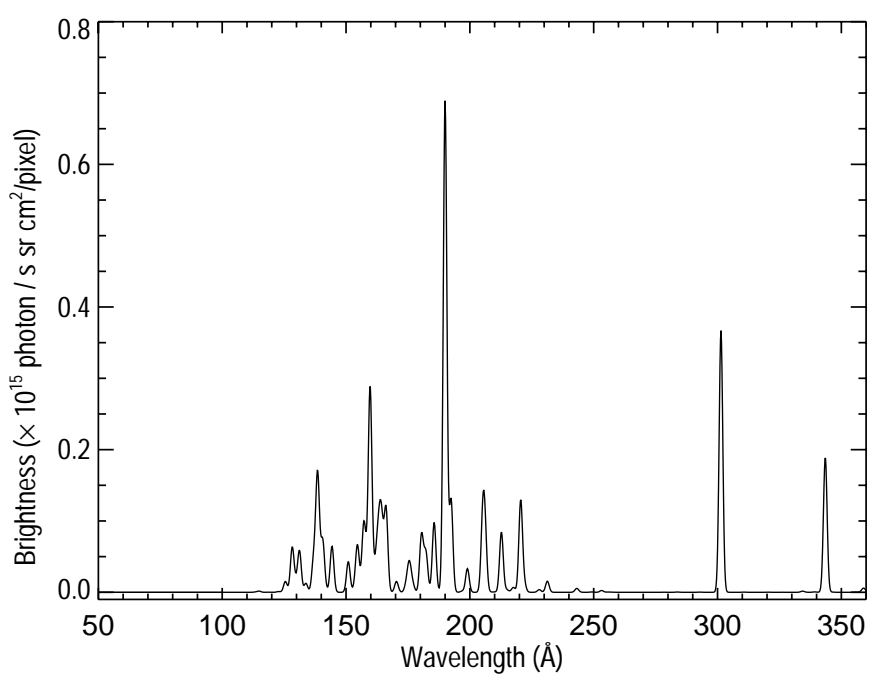

Fig. 6. Synthetic line-integrated time-averaged XUV spectrum of highly ionized calcium in the TEXT tokamak

\section{Results and discussion}

\subsection{Ionization balance}

The LOS-integrated TEXT spectrum was modeled using fractional abundances estimated as described in Sect. 2.3. 
Table 4. Observed and calculated relative line intensities in FTU tokamak

\begin{tabular}{|c|c|c|c|c|}
\hline \multirow[b]{3}{*}{ Ion } & \multirow[b]{3}{*}{$\lambda(\AA)^{\mathrm{c}}$} & \multicolumn{3}{|c|}{ Relative Intensity $^{\mathrm{a}}$} \\
\hline & & \multirow[t]{2}{*}{ Measured } & \multicolumn{2}{|c|}{ Calculated $^{\mathrm{d}}$} \\
\hline & & & HULLAC & CHIANTI \\
\hline \multirow[t]{5}{*}{ Ca XVI } & 154.9 & 14 & 17.8 & 17.7 \\
\hline & 157.8 & 17 & 17.2 & 13.4 \\
\hline & 164.2 & 100 & 100.0 & 100.0 \\
\hline & 167.4 & 40 & 39.8 & 48.0 \\
\hline & 168.9 & 51 & 48.2 & 67.6 \\
\hline \multirow[t]{8}{*}{$\mathrm{CaXV}$} & 161.0 & 100 & 100.0 & 100.0 \\
\hline & 171.6 & 34 & 19.2 & 18.2 \\
\hline & 176.0 & 18 & 12.4 & 12.1 \\
\hline & 176.9 & 46 & 31.7 & 30.4 \\
\hline & 177.3 & 35 & 23.8 & 24.2 \\
\hline & 181.9 & 98 & 97.1 & 95.9 \\
\hline & 182.9 & 28 & 20.1 & 18.3 \\
\hline & 201.0 & 48 & 41.8 & 39.5 \\
\hline \multirow[t]{7}{*}{ Ca XIV } & 153.2 & 13 & 9.0 & 13.5 \\
\hline & 165.3 & 27 & 38.0 & 53.0 \\
\hline & 167.0 & 46 & 51.6 & 67.4 \\
\hline & 183.5 & 15 & 32.7 & 33.9 \\
\hline & 186.6 & 62 & 65.4 & 66.0 \\
\hline & 189.0 & 12 & 10.3 & 14.0 \\
\hline & 193.9 & 100 & 100.0 & 100.0 \\
\hline \multirow[t]{6}{*}{ Ca XIII } & 156.7 & 37 & 34.3 & 34.1 \\
\hline & 159.8 & 17 & 23.6 & 24.5 \\
\hline & 161.7 & 100 & 100.0 & 100.0 \\
\hline & 162.9 & 14 & 18.0 & 18.0 \\
\hline & 164.1 & 23 & 22.9 & 23.0 \\
\hline & 168.4 & 53 & 30.3 & 30.5 \\
\hline
\end{tabular}

${ }^{\mathrm{a}}$ Line intensity, relative to some representative line in each isosequence.

${ }^{\mathrm{b}}$ Labels refer to Fig. 7.

${ }^{\mathrm{c}}$ From Kelly (1987).

${ }^{\mathrm{d}}$ For $T_{\mathrm{e}}$ and $n_{\mathrm{e}}$ values, corresponding to the measured MA location of the ion.

Ionization and recombination rates, used for this calculation, were taken from the recent work of Mazzotta et al. (1998). Their ionization equilibrium curves for the calcium ions differ from those of Arnaud \& Rothenflug 1985, both in MA temperature and ionization fractions. The difference is most pronounced, up to $200 \%$, for the lower ionization stages (Ca XIV - Ca XII) due to improved dielectronic recombination rates. Our calculated calcium ion ground state distributions closely reproduced the measured ones (Lippmann et al. 1987). Using QSS emissivities, we constructed a line-integrated time-averaged synthetic TEXT spectrum (Fig. 6). All lines predicted by HULLAC are shown. This includes not only the $\Delta n=0$ transitions of interest, but also a large number of $\Delta n=1$ and $\Delta n=2$ transitions. These lines are grouped in two domains: $14 \AA$ $\leq \lambda \leq 45 \AA$ and $50 \AA \leq \lambda \leq 100 \AA$. The calculated intensities of these lines are typically a factor of $10^{2}-$ $10^{3}$ less than the $\Delta n=0$ lines. The $50-100 \AA$ domain is covered by GRITS, however, line intensities are below the detector sensitivity limit. As seen in Fig. 6, these lines contribute insignificantly to $\Delta n=0$ line or background intensities. All lines were given a Gaussian shape with the FWHM of GRITS. The agreement between wavelengths from Kelly (1987) and HULLAC is $\leq 1.5 \%$ for most lines, which, however, resulted in different blend patterns (comp. to Fig. 3). Good agreement between synthetic and measured spectra can be considered as indirect validation of the new fractional abundance calculations for calcium.

\subsection{Individual ions}

The calculated relative line intensities for Li I-like to F Ilike calcium ions agree with the measured ones within the stated experimental error in most cases. In comparison with Lippmann et al. (1987), improved atomic rates result in a better agreement between the measured and calculated relative line intensities. Results for individual ions are discussed below. The emphasis is on the beryllium- to nitrogen-like calcium ions, due to their plasma temperature and density diagnostic potential. Calculated density dependence of the level populations is shown in Fig. 7. Both ground and excited level populations are in corona equilibrium at $n_{\mathrm{e}}=10^{9} \mathrm{~cm}^{-3}$. The ground configuration levels approach Boltzmann values at $n_{\mathrm{e}}=10^{10}$ $10^{14} \mathrm{~cm}^{-3}$, and transfer population to the excited $2 \mathrm{p}^{k+1}$ levels at different, $n_{\mathrm{e}}$-dependent, rates. This enables utilization of the $\Delta n=0 \mathrm{E} 1$ line intensity ratios as density diagnostics. Forbidden (M2) transitions between ground levels of CaXv, CaXIV and Ca XIII can also be used as a density diagnostics. These far ultraviolet lines have been observed in a solar active region by Feldman et al. (1998) and used to study physical conditions of the solar corona. Because of the high edge tokamak plasma density $\left(n_{\mathrm{e}} \simeq\right.$ $\left.10^{12} \mathrm{~cm}^{-3}\right)$, collisional quenching of the upper levels of these transitions overtakes radiative decay: both FTU and TEXT plasma edge spectra, recorded by the SPRED and NITS instruments, respectively, indicated no evidence of the forbidden lines. The line intensities, calculated for the edge tokamak densities, are a factor of $10^{2}-10^{3}$ less than those of typical XUV lines, which is beyond the photometric sensitivity limit of the instruments used.

CaXVIII - The only XUV lines identified in solar flare spectrum are $\lambda 302.2$ and $\lambda 344.8$ (see references in Lawson $\&$ Peacock 1984). The lines are populated according to their statistical weights. The discrepancy between calculations and measurements in the TEXT tokamak is attributed to the GRITS calibration uncertainty above $300 \AA$. 


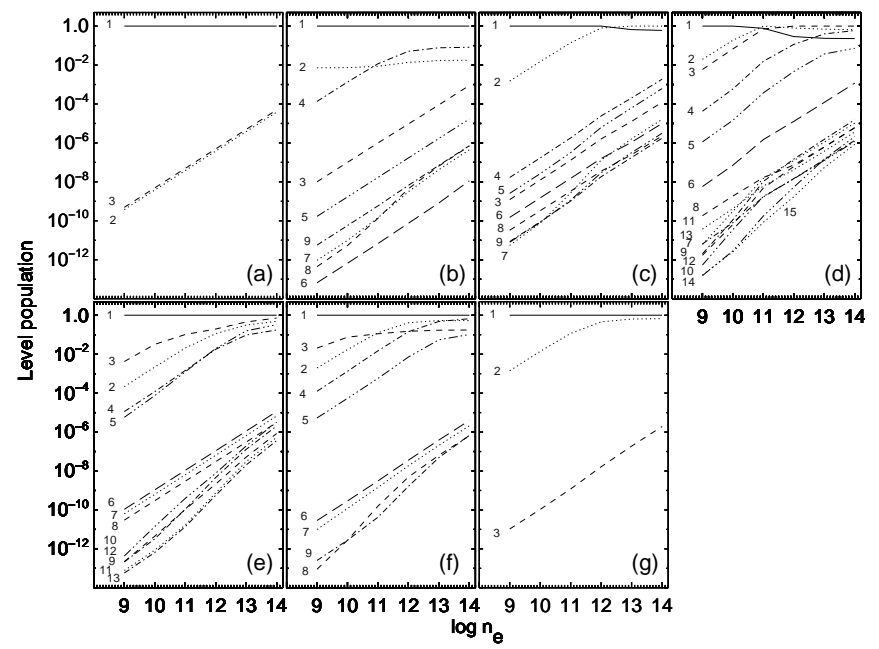

Fig. 7. Modeled relative populations of the $n=2$ levels as a function of electron density at $T_{\mathrm{e}}$ values given in Table 1 (basic model): a) - CaXvIII, b) - CaXVII, c) - CaXVI, d) - CaXV, e) - Ca XIV, f) - Ca XIII, g) - Ca XII. Level populations are normalized to the total population per ion. The level labels are shown in Tables 2 and 3. The levels not shown in Tables 2, 3 are labeled as follows: b) Ca XVII: $6-1 \mathrm{~s}^{2} 2 \mathrm{p}^{2}{ }^{3} \mathrm{P}_{0}$; c) Ca XVI: 3 $\left.-1 \mathrm{~s}^{2} 2 \mathrm{~s} 2 \mathrm{p}^{2}{ }^{4} \mathrm{P}_{1 / 2} ; 4-1 \mathrm{~s}^{2} 2 \mathrm{~s} 2 \mathrm{p}^{2}{ }^{4} \mathrm{P}_{3 / 2} ; 5-1 \mathrm{~s}^{2} 2 \mathrm{~s} 2 \mathrm{p}^{2}{ }^{4} \mathrm{P}_{5 / 2} ; \mathbf{d}\right)$ CaXv: $5-2 \mathrm{~s}^{2} 2 \mathrm{p}^{2}{ }^{1} \mathrm{~S}_{0} ; 6-2 \mathrm{~s} 2 \mathrm{p}^{3}{ }^{5} \mathrm{~S}_{2}$

CaXVII - An example of a persisting disagreement between calculations and solar flare measurements is a long-standing problem with the line intensity ratios of the BeI-like isosequence $R=I\left(2 \mathrm{~s}^{2}{ }^{1} \mathrm{~S}_{0}-\right.$ $\left.2 \mathrm{~s} 2 \mathrm{p}^{1} \mathrm{P}_{1}\right) / I\left(2 \mathrm{~s} 2 \mathrm{p}^{3} \mathrm{P}_{J^{\prime}}-2 \mathrm{p}^{2}{ }^{3} \mathrm{P}_{J^{\prime \prime}}\right), \quad J^{\prime}=1,2$ and $J^{\prime \prime}=1,2$. These intensity ratios are density sensitive (Doschek et al. 1977). The Be I-like neon, magnesium, sulfur, argon and calcium lines have been observed in the solar flares by the NRL Skylab-based S082A spectroheliograph. The calculated line intensity ratios have consistently implied an electron density of $\sim 10^{13} \mathrm{~cm}^{-3}$ and higher, in contrast to the density of $\leq 10^{10}-10^{12} \mathrm{~cm}^{-3}$, derived from other line intensity ratios (Doschek et al. 1977; Bhatia \& Mason 1983; Dufton et al. 1983; McCann et al. 1989; Harra et al. 1992). All six lines have been observed in TEXT. Calculated and measured relative intensities agree within $40 \%$, with the two exceptions of $\lambda 228.7$ and $\lambda 244.0$, which are blended with the FIV lines. Except for the $\lambda 192.9$ and $\lambda 232.8$ lines, all other lines are relatively weak and the inferred densities have larger uncertainties or unrealistic values. We therefore investigated various $\mathrm{CR}$ effects on the $R_{1}=I\left(2 \mathrm{~s}^{2}{ }^{1} \mathrm{~S}_{0}-2 \mathrm{~s} 2 \mathrm{p}^{1} \mathrm{P}_{1}\right) / I\left(2 \mathrm{~s} 2 \mathrm{p}^{3} \mathrm{P}_{1,2}-2 \mathrm{p}^{2}{ }^{3} \mathrm{P}_{1,2}\right)=$ $I(\lambda 192.8) \quad / \quad I(\lambda 232.8)$ ratio. As the number of levels, included in the model, increase, the calculated $I\left(2 \mathrm{~s}^{2}{ }^{1} \mathrm{~S}_{0}-2 \mathrm{~s} 2 \mathrm{p}^{1} \mathrm{P}_{1}\right) / I\left(2 \mathrm{~s} 2 \mathrm{p}^{3} \mathrm{P}-2 \mathrm{p}^{2}{ }^{3} \mathrm{P}\right)$ ratios decrease, due to cascades to the $2 \mathrm{p}^{2}$ levels. This effect is $\leq 20 \%$ as $n=3,4$ levels are added to the model. In particular, the $R_{1}$ ratio was found to be $61(n=2$ only; 10 lowest levels), 49 ( $n=2,3 ; 30$ levels $)$ and
47 ( $n=2,3,4 ; 125$ levels). The ratio, measured in TEXT, is $\sim 51 \pm 5$. Both $\lambda 192.8$ and $\lambda 232.8$ lines are primarily populated by electron impact excitation. The $2 \mathrm{~s} 2 \mathrm{p}^{1} \mathrm{P}_{1}$ level is populated from the ground, whereas the $2 \mathrm{p}^{23} \mathrm{P}$ levels are populated both from the ground and from the $2 \mathrm{~s} 2 \mathrm{p}^{3} \mathrm{P}$ levels. The DWA rates for these transitions, calculated by HULLAC, are within $\leq 15 \%$ of the R-matrix rates calculated by Dufton et al. (1983), for the $400-900 \mathrm{eV}$ temperature range (with one exception of $2 \mathrm{~s}^{2}{ }^{1} \mathrm{~S}_{0}-2 \mathrm{~s} 2 \mathrm{p}^{1} \mathrm{P}_{1}$, for which the HULLAC rate is $\simeq 35 \%$ smaller than the rate from Dufton et al. 1983). The detailed model was used for Ca XVIII, Ca XVII and Ca XVI, to account for possible non-steady state contributions. The model was constructed for both tokamak and ionization equilibrium temperatures. Direct ionization is found to be the dominating process, the autoionization flux originating from the $1 \mathrm{~s} 2 \mathrm{l}^{2}$ levels of CaXVI is negligible, and the population flux due to inner-shell ionization from $2 \mathrm{~s}^{2} 2 \mathrm{p}$ and $2 \mathrm{~s} 2 \mathrm{p}^{2}$ levels of CaXVI to $2 \mathrm{~s} 2 \mathrm{p}^{3} \mathrm{P}$ levels of CaXVII is several orders of magnitude less than the flux to the ground state. Significant departure from ionization equilibrium is required to populate the $2 \mathrm{~s} 2 \mathrm{p}{ }^{3} \mathrm{P}$ levels through inner-shell ionization (Feldman et al. 1992). Our calculations indicate that if $n_{\mathrm{CaXVII}} / n_{\mathrm{CaXVI}}=1: 10$, at $n_{\mathrm{e}}=310^{11} \mathrm{~cm}^{-3}$ up to $50 \%$ of the total population of $2 \mathrm{~s} 2 \mathrm{p}^{3} \mathrm{P}$ levels is due to inner-shell ionization, and the $R_{1}$ ratio is practically the same as at $n_{\mathrm{e}}=310^{13} \mathrm{~cm}^{-3}$ at ionization equilibrium conditions. We conclude, therefore, that the $R_{1}$ ratio should be a reliable density diagnostics in the $n_{\mathrm{e}}$ range of $10^{10}-10^{14} \mathrm{~cm}^{-3}$.

Intensity ratio of two $\Delta n=0$ lines of the same ion can be used as a temperature diagnostic due to the temperature dependence of the excitation rate coefficients, if the separation of the upper levels of these lines $\Delta E$ is much less than $k T_{\mathrm{e}}$, as in the case of the resonant and intercombination lines of Be Ilike calcium, $R_{2}(\mathrm{CaXVII})=I\left(2 \mathrm{~s} 2 \mathrm{p}^{3} \mathrm{P}_{1}-2 \mathrm{~s}^{2}{ }^{1} \mathrm{~S}_{0}\right)$ $/ I\left(2 \mathrm{~s} 2 \mathrm{p}^{1} \mathrm{P}_{1}-2 \mathrm{~s}^{2}{ }^{1} \mathrm{~S}_{0}\right) \quad=\quad I(\lambda 371.0) / I(\lambda 192.8)$ $(\Delta E \simeq 32 \mathrm{eV})$. Figure 8 presents the calculated $R_{2}$ as a function of logarithmic electron temperature in $\mathrm{K}$. The lines have been observed in the solar flare on 09 August 1973 by the Skylab SO82A. The ratios are $0.05-0.08$ (measured by Doschek et al. 1977) and $0.027-0.058$ (re-measured by Dufton et al. 1983). Our calculations yield the logarithmic temperature between 5.9 and 7.2, derived from the latter measurement. The CaXVII ionization equilibrium temperature is $\log T_{\mathrm{e}}=6.74$ (Arnaud \& Rothenflug 1985) and 6.78 (Mazzotta et al. 1998). McCann et al. (1989), using the SO82A observational data for the same flare, measured the $R_{2}$ ratio for the ions $\mathrm{S}$ XIII and $\mathrm{ArXV}$, and derived the logarithmic temperatures of $\sim 6.5$ (sulfur), 6.0 and 6.45 (argon). These temperatures are within $20 \%$ of the ionization equilibrium temperatures of sulfur and argon, respectively. The temperature, inferred from the $I(\lambda 371.0) / I(\lambda 192.8)$ ratio, is consistent with the 
latter measurements and is reasonable for solar flares, although clearly much higher accuracies in the intensity measurements are needed to utilize this type of line ratio techniques.

Relative line intensities, calculated using the CHIANTI database, are close to the HULLAC calculation and consistent with the experimentally inferred intensities.

CaXVI - The blending problem is especially aggravated in the 150 - $170 \AA$ domain, densely populated by CaXIV, CaXV and Ca XVI lines. We note that our calculations did not reconcile the discrepancy between measured and calculated intensities of the $\lambda 208.6$ and $\lambda 224.6$ lines (upper levels $2 \mathrm{p}^{2}{ }^{2} \mathrm{D}$ ). Lippmann et al. (1987) attributed them to the fact that their model did not include collisional excitation between $2 \mathrm{~s} 2 \mathrm{p}^{2}{ }^{2} \mathrm{D}$ levels and the $2 \mathrm{p}^{3}{ }^{2} \mathrm{D}$ levels, which resulted in overestimation of the upper level populations. Recent calculations of Keenan et al. (1998), which used R-matrix collision rates and included the $2 \mathrm{p}^{3}$ levels as well, demonstrate a similar trend. HULLAC excitation rates between these terms are comparable to the excitation rates from the ground. According to our calculations, however, these excitation processes become noticeable ( $\geq 10 \%$ in the $2 \mathrm{~s} 2 \mathrm{p}^{2}{ }^{2} \mathrm{D}$ level populations) only at $n_{\mathrm{e}} \geq 5$ $10^{14} \mathrm{~cm}^{-3}$. The $\lambda 150-170 \AA$ lines and the $\lambda 208.6$ and $\lambda 224.6$ lines were recorded from different TEXT discharges, which could have explained the difference. The relative line intensities of the $\lambda 150-170 \AA$ lines from both TEXT and FTU datasets are in good agreement with HULLAC calculations. The ratio $R=I(\lambda 224.6) / I(\lambda 208.6)=1.3$, recorded in TEXT, also agrees well with HULLAC prediction of 1.25. Keenan et al. (1998) pointed out that CHIANTI database gives abnormally low intensity of the latter ratio. Whereas this was due to a missing piece of data and has been corrected in v. 2.0 (Landi et al. 1999), the CHIANTI relative intensities still differ from our measurements and HULLAC calculations. The measured branching ratios of the lines originating from the $2 \mathrm{~s} 2 \mathrm{p}^{2}{ }^{2} \mathrm{P}_{1 / 2,3 / 2}$ levels agree well with computations.

Several Ca XVI line pairs are electron density sensitive, and they have been used by Dere et al. (1979) and Keenan et al. (1998) in application to solar flare diagnostics. In addition to the mentioned $R$ ratio, the intensity ratios of $\lambda 154.9, \lambda 157.8, \lambda 164.2, \lambda 168.9$ to $\lambda 208.6$ are also density sensitive. The $R$ ratio, however, seems to be a more reliable diagnostics, since the lines are close and subject to blends to a lesser degree.

CaXV - As in the case of boron-like calcium, many of the lines are blended, and in some cases $(150-170 \AA)$ it was difficult to measure the brightnesses accurately. The $\lambda 208$ blend was separated to the three components $\lambda 208.3, \lambda 208.7$ (CaXV) and $\lambda 208.6$ (CaXVI) using our model. Ten out of fourteen recorded lines in TEXT

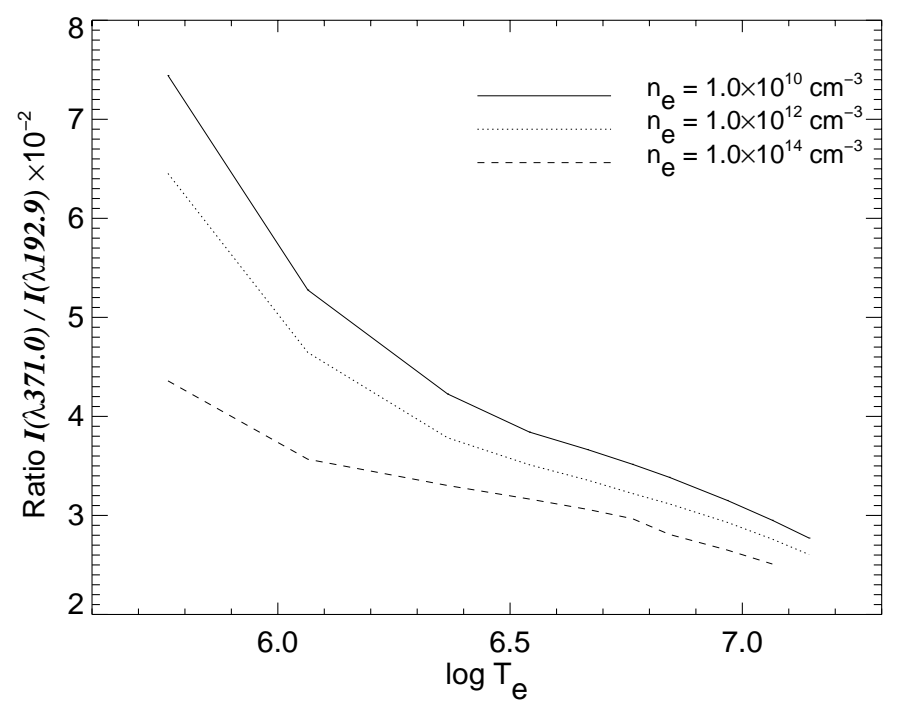

Fig. 8. Predicted Ca XVII line intensity ratio $R_{2}=I\left(2 \mathrm{~s} 2 \mathrm{p}^{3} \mathrm{P}_{1}-\right.$ $\left.2 \mathrm{~s}^{2}{ }^{1} \mathrm{~S}_{0}\right) / I\left(2 \mathrm{~s} 2 \mathrm{p}^{1} \mathrm{P}_{1}-2 \mathrm{~s}^{2}{ }^{1} \mathrm{~S}_{0}\right)=I(\lambda 371.0) / I(\lambda 192.8)$ as a function of electron temperature

and most of the lines recorded at FTU agree within the experimental error with the measurements. Both HULLAC and CHIANTI models include the important $2 \mathrm{p}^{4}$ configuration. The radiative cascade effects are small: line intensities, calculated using HULLAC $(n=2$, $n=2,3$, and $n=2,3,4$ models) and CHIANTI data ( $n=2$ only), differ by $\leq 5 \%$. Several line ratios have been used to infer electron densities in solar flares (Dere et al. 1979; Keenan et al. 1992, and references therein). Present HULLAC calculations do not improve upon density estimates previously published.

CaXIV - HULLAC calculations agree within the error of measurements with both TEXT and FTU datasets for the majority of the lines. The effect of cascades from $n=3$ and $n=4$ levels is weak, $\leq 10 \%$. Inclusion of the $2 \mathrm{p}^{5}$ levels affects the $2 \mathrm{~s}^{2} 2 \mathrm{p}^{3}-2 \mathrm{~s} 2 \mathrm{p}^{4}$ relative line intensities insignificantly $(\leq 5 \%)$. CHIANTI intensities are in overall good agreement with the measurements, however some line intensities disagree up to a factor of two. In the same time, there is a close agreement between several measured branching ratios and the branching ratios from HULLAC and CHIANTI. Diagnostics potential of CaXIV lines includes the density-sensitive intensity ratios of the lines, originating from $2 \mathrm{~s} 2 \mathrm{p}^{4}{ }^{4} \mathrm{P}$ to the lines, originating from $2 \mathrm{~s} 2 \mathrm{p}^{4}{ }^{2} \mathrm{~S},{ }^{2} \mathrm{P},{ }^{2} \mathrm{D}$ (Feldman et al. 1980). The line intensity ratios of $\lambda 134, \lambda 165, \lambda 167, \lambda 189$ to $\lambda 194$ are density sensitive up to $n_{\mathrm{e}} \simeq 10^{14} \mathrm{~cm}^{-3}$. Although $\lambda 194$ is blended with a very strong $\lambda 193$ CaxviI line, it is possible to accurately separate them. The density predictions for TEXT, for example, based on the intensity ratio of $\lambda 165$ and $\lambda 189$ to $\lambda 194$ are within $20 \%$ of the independently measured value. Three most intense XUV lines $(\lambda 183.5, \lambda 186.6$ and $\lambda 193.9)$ have been identified in the full Sun spectra (Behring et al. 1972), and recently in 
the transition region (Brosius et al. 1998).

CaXIII - Both HULLAC and CHIANTI calculations agree well with the TEXT and FTU datasets. Several line intensity ratios, e.g. $\lambda 131, \lambda 160$ to $\lambda 164$, can be used as density diagnostics in the range between $10^{10}$ and $10^{13} \mathrm{~cm}^{-3}$. The Ca XIII XUV lines have not been observed in solar plasmas.

CaxII - The two XUV lines ( $\lambda 141.0$ and $\lambda 147.3)$ have been identified in the full Sun spectra (Behring et al. 1972). The lines share the same upper level $2 \mathrm{~s} 2 \mathrm{p}^{6}{ }^{2} \mathrm{~S}_{1 / 2}$ and their intensity ratio is simply a ratio of the transition probabilities, known with fairly high accuracy. As follows from Fig. 5, the $\lambda 141$ is a blend, which is the reason for a $20 \%$ difference between the measured and calculated relative intensities.

\section{Conclusions}

Two CR models of highly ionized calcium have been benchmarked in this work by comparing their predictions to the line intensities, measured with an absolutely calibrated grazing incidence spectrometer in two tokamak experiments. The CR models are based on ab initio HULLAC and CHIANTI atomic data, which are the state of the art in atomic structure calculations. The HULLAC models are in a good agreement with most measured lines of lithium-like to fluorine-like calcium. CHIANTI predictions for several CaXVI and CaXIV lines are inconsistent with our measurements and HULLAC calculations. It is shown that for the tokamak, as well as for the solar flare plasma conditions, collisional-radiative models which include $n=2$ and $n=3$ configurations are adequate to predict L-shell line intensities. At these plasma conditions, radiative or collisional cascades from $n=4,5$ levels are generally insignificant, and quasi-steady state approach applies well. With the present state of atomic calculations, likely explanations for large discrepancies with experimental data are transient or kinetic effects, not the atomic data quality. Total ionization and recombination rates which are used by Mazzotta et al. (1998) for a new calcium fractional abundances calculation, have been used for a calcium L-shell spectrum simulation. A synthetic lineintegrated spectrum which includes over two hundred lines in the range $50-360 \AA$, predicted by HULLAC, closely reproduces the spectrum recorded at the TEXT tokamak. Density predictions based on line ratios of beryllium-, boron-, carbon-, nitrogen-, and oxygen-like calcium are compared with independent density measurements. Good agreement is found in the cases with minimal experimental uncertainties.

All computational data are available in electronic form upon request.
Acknowledgements. The authors would like to acknowledge the TEXT and FTU tokamak teams. This work was supported by U.S. DoE Grant DE-FG02-86ER53214 at JHU and Contract No. W-7405-ENG-48 at LLNL.

\section{References}

Aggarwal K.M., Berrington K.A., Keenan F.P., 1990, ApJS 72, 815

Aggarwal K.M., 1992, ApJS 80, 453

Aggarwal K.M., Hibbert A., Keenan F.P., Norrington P.H., 1997, ApJS 108, 575

Andreani R., 1993, Fusion Eng. Des. 22, 129

Arnaud M., Rothenflug R., 1985, AAS\&S 60, 425

Baliyan K.S., Bhatia A.K., 1994, J. Phys. B 27, 4281

Bar-Shalom A., Klapish M., Oreg J., 1988, Phys. Rev. A, 38, 1773

Bell R.E., Finkenthal M., Moos H.W., 1981, Rev. Sci. Instrum. 52,1806

Behring W.E., Cohen L., Feldman U., 1972, ApJ 175, 493

Bhatia A.K., Mason H.E., 1983, A\&AS 52, 115

Bhatia A.K., Feldman U., Seely J.F., 1986, At. Data Nucl. Data Tab. 35, 449

Bhatia A.K., Doschek G.A., 1993, At. Data Nucl. Data Tab. 53,195

Brickhouse N.S., Raymond J.C., Smith B.W., 1995, ApJS 97, 551

Brosius J., Davila J., Thomas R., Thompson W., 1994, ApJ 425,343

Brosius J.W., Davila J.M., Thomas R.J., 1998, AS\&S 119, 255

Dere K.P., Mason H.E., Widing K.G., Bhatia A.K., 1979, A\&AS 40, 341

Dere K.P., Landi E., Manson H.E., Monsignori-Fossi B.F., Young P.R., 1997, A\&AS 125, 149

Doschek G.A., Feldman U., Dere K.P., 1977, A\&A 60, L11

Dufton P.L., Kingston A.E., Doyle J.G., Widing K.G., 1983, MNRAS 205, 81

Dufton P.L., Kingston A.E., Scott N.S., 1983, J. Phys. B 16, 3053

Feldman U., Doschek G.A., Cheng C.C., Bhatia A.K., 1980, J. Appl. Phys. 51, 190

Feldman U., Doschek G.A., Seely J.F., 1988, J. Opt. Soc. Am. B 5, 2237

Feldman U., Laming J.M., Mandelbaum P., Goldstein W.H., Osterheld A., 1992, ApJ 398, 692

Feldman U., Mandelbaum P., Seely J.F., Doschek G.A., Gursky H., 1992, ApJS 81, 387

Feldman U., Curdt W., Doschek G.A., Schuhle U., Wilhelm K., Lemaire P., 1998, ApJ 503, 467

Finkenthal M., Lippmann A.S., Huang L.K., et al., 1986, J. Appl. Phys. 59, 3644

Finkenthal M., Yu T.L., Lippmann S., et al., 1987, ApJ 313, 920

Fonk R.J., 1982, Appl. Opt. 21, 2115

Gentle K.W., 1981, Nucl. Tech. Fus. 1, 479

Harra L.K., Keenan F.P., Widing K.G., Conlon E.S., 1992, in UV and X-ray Spectroscopy of Laboratory and Astrophysical Plasma

Harrison R.E., et al., 1995, Solar Phys. 162, 233

Hodge W.L., Stratton B.C., Moos H.W., 1984, Rev. Sci. Instr. 55,16 
Horton W., Rowan W., 1994, Phys. Plasmas 1, 901

Huang L.K., Lippmann S., Yu T.L., et al., 1987, Phys. Rev. 35,2919

Hulse R.A., 1983, Nucl. Tech. Fus. 3, 259

Keenan F.P., Conlon E.S., Foster V.J., Aggarwal K.M., Widing K.G., 1992, ApJ 401, 411

Keenan F.P., Pinfield D.J., Woods V.J., Reid R.H.G., Conlon E.S., Pradhan A.K., Zhang H.L., Widing K.G., 1998, ApJ 503, 953

Kelly R.L., 1987, J. Phys. Chem. Ref. Dat. 16, No. 1

Klapisch M., 1971, Comp. Phys. Commun. 2, 269

Klapisch M., Schwob J.L., Fraenkel B.S., Oreg J., 1977, J. Opt. Soc. Am. 67, 148

Landi E., Landini M., 1997, A\&A 327, 1230

Landi E., Landini M., Dere K.P., Young P.R., Mason H.E., 1999, A\&AS 135, 339

Lang J., 1996, Phys. Scr. T65, 115

Lawson K.D., Peacock N.J., 1984, A\&AS 58, 475

Lippmann S., Finkenthal M., Huang L.K., Moos H.W.,
Stratton B.C., Yu T.L., Bhatia A.K., Hodge W.L., 1987, ApJ 316, 819

Lotz W., 1968, Z. Phys. 216, 241; Lotz W., 1970, 232, 101

Mason H.E., 1991, Adv. Space Res. 11 (1), 293

Mason H.E., Monsignori Fossi B.C., 1994, ARA\&A 6, 123

Mason H.E., Young P.R., Pike D.D., et al., 1997, Sol. Phys. 170, 143

McCann S.M., Keenan F.P., Widing K.G., 1989, J. de Phys. 50, C1-571

Mazzotta P., Mazzitelli G., Colafrancesco S., Vittorio N., 1998, A\&A 133, 403

Phillips K.J.H., Bhatia A.K., Mason H.E., Zarro D.M., 1996, ApJ 466, 549

Stratton B.C., Moos H.W., Suckewer S., et al., 1985, Phys. Rev. A 31, 2534

Terry D.R., Rowan W., Conolly W.C., Leung W.K., 1983, in Proc. 10th Symp. Fusion Energy 14, 959

Young P.R., Landi E., Thomas R.J., 1998, A\&A 329, 291

Zhang H.L., Pradhan A.K., 1994, Phys. Rev. A 50, 3105 